Revue interdisciplinaire des études canadiennes en

France

$80 \mid 2016$

Le Canada et la Grande Guerre

\title{
Bien-être et pratiques culturelles. Comparaison du cas des Inuit du Nunavut au Canada et des Quichuas du Napo en Amazonie équatorienne
}

Wellbeing and cultural practices: a comparison between the Nunavut Inuit in Canada and the Napo Quichuas in equatorial Amazonia

\section{Simon Fleury}

\section{CpenEdition}

Journals

Édition électronique

URL : http://journals.openedition.org/eccs/732

DOI : $10.4000 /$ eccs.732

ISSN : 2429-4667

Éditeur

Association française des études canadiennes (AFEC)

Édition imprimée

Date de publication : 1 juin 2016

Pagination : 203-218

ISSN : 0153-1700

Référence électronique

Simon Fleury, «Bien-être et pratiques culturelles. Comparaison du cas des Inuit du Nunavut au Canada et des Quichuas du Napo en Amazonie équatorienne », Études canadiennes / Canadian Studies [En ligne], 80 | 2016, mis en ligne le 01 juin 2017, consulté le 21 avril 2019. URL : http:// journals.openedition.org/eccs/732 ; DOI : 10.4000/eccs.732 


\title{
BIEN-ETRE ET PRATIQUES CULTURELLES. COMPARAISON DU CAS DES INUIT DU NunAVUT AU CANADA ET DES QUiCHUAS DU NAPO EN AMAZONIE EQUATORIENNE
}

\author{
Simon FLEURY \\ (Université de Poitiers \\ Université de Nanterre Paris La Défense)
}

\begin{abstract}
L'article aborde le thème de la santé mentale et de la résilience autochtones au travers d'une étude comparative entre Inuit du Nunavut au Canada et Quichuas du Napo en Équateur. Il essaye d'apporter un éclairage pour comprendre les processus historiques pouvant mener à un fort taux de suicide chez les jeunes Inuit. L'existence de liens entre pratiques culturelles et santé mentale et également entre pratiques culturelles et reconnaissance sont interrogés. L'objectif de l'article est de proposer des nouvelles pistes de réflexions dans le domaine du bien-être autochtone.

The article is about native mental health and resilience. It is a comparative study between the Inuit of Nunavut in Canada and the Quichuas of Napo in Ecuador. It offers to understand the historical process leading to a high rate of suicide especially among the young Inuit. The existence of links between cultural practices and mental health and then between cultural practices and recognition are investigated. The article tends to offer new reflections in the field of native wellbeing.
\end{abstract}

\section{Introduction}

Toutes les communautés autochtones ne présentent pas des taux de suicide, d'alcoolisme et d'addiction élevés indiquant une santé mentale détériorée. Cependant, les plus hauts taux de ces mêmes phénomènes se retrouvent généralement au sein des populations autochtones (GOEBERT 2014 : 159). Les taux de suicide, la consommation d'alcool et de drogues, des comportements étiquetés comme dépressifs et témoignant d'une santé mentale instable atteignent chez certaines populations des proportions telles, que l'on parle aujourd'hui d'une nouvelle forme d'épidémie ${ }^{1}$. La santé mentale apparaît donc pour les autochtones comme l'un des défis centraux, qui relève, selon la définition donnée par l'Organisation Mondiale de la Santé, du « bien-être »².

\footnotetext{
${ }^{1}$ Notamment Padma Suramala, experte médico-légal du Nunavut travaillant sur la prévention $\mathrm{du}$ suicide (CBC News, «Padma Suramala, Nunavut's coroner, calls inquest into suicides », Jan 16, 2014 11:54 AM). Egalement Marie Wilson (Déléguée de la Commission sur la Vérité et la Réconciliation)

${ }^{2}$ Site internet officiel de l'OMS, 2015. Préambule à la Constitution de l'Organisation mondiale de la Santé, tel qu'adopté par la Conférence internationale sur la Santé, New York, 19-22 juin 1946;
} 


\section{Simon FLEURY}

Parallèlement à cette réalité, les revendications autochtones et l'obtention d'une reconnaissance à différents degrés (politique, territorial et identitaire) au sein des États-nation où se situent les groupes et au niveau international par le biais de 1'ONU (Organisation des Nations Unies) et de l'OIT (Organisation Internationale du Travail) ${ }^{3}$, connaissent une évolution rapide depuis les années 1960. Les groupes autochtones, souvent à l'origine de mouvements sociaux et contestataires, ont su s'organiser formellement en s'appropriant des instruments politiques. Les revendications et projets autochtones s'inscrivent dans une approche axée sur le local et le territoire, défendant l'idée de l'autodétermination des peuples et d'une plus grande autonomie. Elles s'appuient sur des arguments identitaires et historiques. L'identité est donc un deuxième sujet central chez les autochtones. Mais quel est le lien entre identité et bien-être ? La reconnaissance identitaire assure-t-elle le bien-être?

En 1999, suite à de nombreuses négociations, le Canada créait en collaboration avec les Inuit le territoire du Nunavut doté d'un gouvernement public. Les accords sur la création du territoire du Nunavut prévoient que la fonction publique soit représentative de la population du territoire majoritairement Inuit: c'est-à-dire composée à hauteur de $85 \%$ d'Inuit $^{4}$ (BERGER 2006 :14-15; MORIN 2003 : 7). En 1998 puis en 20085, l'Équateur reconnaissait au niveau constitutionnel le caractère plurinational du pays en conférant aux différents groupes autochtones qui le composent des droits spécifiques sur leur territoire, sans cependant faire référence à la création de gouvernements publics décentralisés.

Le Nunavut représente un exemple au niveau mondial d'un projet autochtone sur un territoire défini conjointement avec le Canada. L'obtention d'une reconnaissance telle et la possibilité de s'organiser, grâce à un

signé le 22 juillet 1946 par les représentants de 61 Etats. 1946; (Actes officiels de l'Organisation mondiale de la Santé, ${ }^{\circ} .2$, p. 100) et entré en vigueur le 7 avril 1948.

${ }^{3}$ Déclaration de l'ONU sur les droits des peuples autochtones (13 septembre 2007) et Convention $\mathrm{n}^{\circ} 169$ relative aux peuples indigènes et tribaux (1989).

${ }^{4}$ Ce n'est cependant pas le cas. A l'heure actuelle, on estime que le gouvernement du Nunavut est composé à $45 \%$ d'Inuit seulement; $15 \%$ dans les postes décisionnels. Thomas Berger, (1 mars 2006), "Le projet Nunavut", Rapport final du conciliateur, Accords sur les revendications territoriales du Nunavut. Négociations du contrat de mise en œuvre pour la deuxième période de planification 2003-2013.

${ }^{5}$ En 1998, une nouvelle constitution reconnaissait la diversité ethnique du pays. La constitution de 2008 renforcera la reconnaissance en utilisant le terme de plurinationalité et en introduisant le concept du Sumak Kausay pour garantir à la nature des droits au même titre que l'être humain. 
gouvernement public au sein d'un territoire, laissent supposer un épanouissement certain des Inuit du Nunavut. Pourtant, le Nunavut est également un territoire ayant l'un des taux de suicide les plus élevés chez les populations autochtones, principalement chez les jeunes de 14 à 24 ans. En 2011, le taux de suicide était de 110 pour 100.000 habitants au Nunavut, soit un taux environ 10 fois supérieur à la moyenne canadienne de 10,8 pour 100.000 habitants la même année (HICKS 2009: 2). Les Quichuas du Napo en Amazonie équatorienne ne possèdent pas de système de gouvernance aussi « abouti » que celui du Nunavut, bien qu'ils s'organisent au niveau politique par le biais de confédérations ${ }^{6}$. Les problèmes d'alcool sont présents, mais on ne recense pas de taux de suicide anormalement élevé.

Comment expliquer un taux de suicide plus important chez les Inuit du Nunavut que chez les Quichuas du Napo malgré la création du Nunavut en 1999, censé conjuguer le «meilleur des deux mondes », c'est à dire la société occidentale et le monde autochtone, pour un mieux-être culturel et social (MAGORD 2005 : 248)? L'identité et le bien-être étant deux thèmes au centre des problématiques autochtones, quelles sont leur place dans le projet du Nunavut au Canada et le projet d'État plurinational en Équateur?

Notre article ne prétend pas apporter de résultats mais souhaite proposer des pistes de réflexion, au travers de l'étude des productions scientifiques, sur le sujet de la santé mentale autochtone et des observations faites sur le terrain équatorien. Une comparaison des deux contextes n'ayant jamais été entreprise auparavant, notre recherche espère apporter un éclairage nouveau sur l'originalité des problématiques Inuit et Quichua. Cet article se penche davantage sur la question Inuit en optant pour une démarche comparatiste selon le modèle référentiel (BOUCHARD 2000: 37-75). Ce dernier consiste à prendre une des unités de comparaison comme référent (ici les Inuit du Nunavut). La comparaison s'élabore en fonction de cette unité. Le détour par une ou plusieurs autres unités (ici les Quichuas du Napo en Équateur) a pour but de mieux connaître l'unité de référence.

\footnotetext{
${ }^{6}$ Au niveau local la FONAKIN (Federacion De Organizaciones De La Nacionalidad Kichwa De Napo); au niveau régional la GONOAE (Gobierno de las Naciones Originarias de la Amazonia Ecuatoriana, anciennement CONFENIAE); au niveau national la CONAIE (Confederacion de las Nacionalidades Indigenas del Ecuador).
} 


\section{Simon FLEURY}

\section{Les chocs historiques}

Les différents processus de colonisation et leur étude ont amené à identifier trois chocs qu'ont connus les populations autochtones: les chocs biologique, structurel et identitaire. Le choc biologique se décrit par les fortes chutes démographiques qu'ont connues la plupart des groupes, lesquelles furent provoquées suite au contact avec des maladies, face auxquelles les systèmes immunitaires ne savaient pas réagir (DELÂGG et TRUDEL 1991: 7, WESLEY-ESQUIMAUX 2007). Le deuxième choc, structurel, découle des ruptures de l'organisation sociale interne et des logiques culturelles venant équilibrer la vie quotidienne. Le choc identitaire enfin, est perçu comme la remise en question existentielle des définitions identitaires collective et individuelle des groupes colonisés. Ces trois chocs nous amènent à nous interroger sur les impacts au niveau collectif qu'ils peuvent encore avoir aujourd'hui et donc à les percevoir comme des déterminants sociaux de la santé mentale autochtone. Duran et Duran décrivent les effets de la colonisation et du colonialisme comme une «blessure de l'âme» qui serait «au cœur d'une grande partie de la souffrance subie par les peuples autochtones pendant plusieurs siècles » (DURAN et DURAN 1995: 24). Nous nous intéresserons plus particulièrement aux chocs structurel et identitaire.

Sans chercher à tout expliquer au travers du filtre de ces trois chocs, nous ne pouvons négliger l'importance qu'ils occupent de nos jours dans les dynamiques sociales des Quichuas et des Inuit. Certaines logiques propres à l'Histoire perdurent dans la façon dont les Quichuas et Inuit ont de percevoir les rapports de pouvoir, et dans la façon dont les pays dans lesquels ils se situent, respectivement l'Équateur et le Canada, ont de penser leurs politiques de reconnaissance. Comme le précise Wesley-Esquimaux (2007:7), des mémoires collectives du traumatisme, encodées par les autochtones il y a quelques siècles, se retrouvent dans les recueils de la mémoire culturelle et sont, aujourd'hui, récupérées et réclamées. De plus, le discours politique dominant a tendance à maintenir la marginalité des peuples autochtones, en perpétuant le caractère singulier de ce que ces peuples ont vécu.

Il est tout de même important de noter que, malgré ces trois chocs, les théories de l'assimilation, du «melting pot» (en Amérique du Nord) et du «métissage » (mestizaje, en Amérique Latine), selon lesquelles les différences culturelles allaient s'estomper, ne se sont pas vérifiées (LACROIX, $2013: 3$ ). De part et d'autre de l'Amérique, dans des contextes souvent très différents, les 
groupes autochtones se sont organisés socialement, puis politiquement, pour revendiquer une reconnaissance identitaire, territoriale et politique.

\section{Rebondir après un traumatisme dans un contexte de mondialisation et de mutations rapides des modes de vie}

Le regain de revendications autochtones depuis les années 1960 montre que les groupes autochtones font preuve de résilience, entendue ici dans une perspective psychosociale. Selon Cyrulnik, la résilience est le «processus biologique, psychoaffectif, social et culturel qui permet un nouveau développement après un traumatisme psychique »(CYRULNIK, 2012: 8). Loin de disparaître, les Inuit et les Quichuas ont cherché à proposer un nouveau projet. Quelles stratégies de résilience ont-ils déployées? L'obtention d'une reconnaissance politique comme celle du Nunavut est-elle la clé de la résilience ? Quelles limites ces stratégies ont-elles connues ? Pourquoi malgré la création du territoire du Nunavut, les Inuit connaissent-ils une santé mentale dégradée?

\section{La santé mentale}

Nous nous intéressons à la définition de la santé mentale donnée par l'OMS en référence, cependant nous précisons que la perception de la santé et du bien-être varient en fonction des cultures. La santé mentale fait l'objet d'un large éventail d'activités qui relèvent directement ou indirectement du «bienêtre », tel qu'il figure dans la définition de la santé établie par l'OMS comme étant « un état de complet bien-être physique, mental et social, et [qui] ne consiste pas seulement en une absence de maladie ou d'infirmité ». La santé mentale englobe la promotion du bien-être, la prévention des troubles mentaux, le traitement et la réadaptation des personnes atteintes de ces troubles ${ }^{7}$.

Cette définition illustre les liens existant entre santé mentale et entourage socioculturel, à l'échelle familiale ou sociétale. La santé mentale n'est pas la simple absence de maladie en des termes somatiques, elle se situe à la croisée de facteurs multiples. Parmi ces facteurs, nous nous intéressons plus particulièrement au rôle des pratiques culturelles, décrites comme centrales par

\footnotetext{
${ }^{7}$ OMS, 2015, préambule à la Constitution de l'Organisation mondiale de la Santé, tel qu'adopté par la Conférence internationale sur la Santé, New York, 19-22 juin 1946; signé le 22 juillet 1946 par les représentants de 61 Etats. 1946; (Actes officiels de l'Organisation mondiale de la Santé, nº. 2, p. 100) et entré en vigueur le 7 avril 1948.
} 


\section{Simon FLEURY}

les Inuit et les Quichuas dans l'expression de leur identité. Nous abordons les pratiques culturelles comme une expérience favorisant les liens sociauxculturels d'un groupe (CYRULKIK et JORLAN 2014). En somme, nous identifions l'expérience culturelle comme une activité menant à un bien-être, dès lors qu'elle est considérée comme partie intégrante de l'identité vécue ou revendiquée.

\section{Quelle vision de la santé?}

Il existe autant de définitions de la santé que de cultures différentes parce que chaque peuple développe un concept du bien-être dérivé de sa propre logique interne et de sa théorie des connaissances. Cependant, selon Alderete (2004: 20), les cultures indigènes ont quelques éléments communs et unificateurs. Le paradigme biomédical occidental considère que le corps, l'esprit et la société sont des éléments compartimentés et que chacun peut se comprendre indépendamment de l'autre. Les systèmes autochtones de soins se basent sur un concept holistique de la santé selon lequel le bien-être se perçoit comme l'harmonie qui existe entre les personnes, les communautés et l'univers qui les entourent. Ce bien-être dépend donc de la santé de la nature, de l'adhésion à des normes sociales et du respect des obligations morales. Alderete oppose la vision compartimentée occidentale du bien-être avec celle holistique de la vision autochtone. Par effet d'identisation et d'identification, une identité autochtone semble se dessiner au travers de la définition du bien-être. Dès lors, il est pertinent de se demander dans quelle mesure le processus de sédentarisation a perturbé ces trois points chez les Inuit et les Quichuas.

\section{Ruptures et continuités dans les modes de vie et santé mentale}

Les Quichuas et les Inuit ont connu, à une période et une vitesse différentes, une sédentarisation contrainte. A partir des années 1950, le Canada a mis en place une politique de relogement et de relocalisation des populations du Nord. Les populations Inuit de l'Arctique canadien ont été déplacées pour créer des villages et communautés. Le but premier n'était pas de venir en aide aux Inuit qui subissaient alors des épidémies de tuberculose et une famine importante, mais d'occuper de façon stratégique les territoires situés au-dessus du $60^{\text {ème }}$ parallèle pour y assoir la souveraineté canadienne, mise à mal dans un contexte de Guerre froide (PELAUDEIX 2008 :161-176). Jusqu'alors, la politique canadienne était de laisser les Inuit vivre de façon nomade, c'est-àdire en se déplaçant en fonction des ressources animales disponibles et des saisons. 
Les Quichuas du Napo avaient une vie semi-nomade. Ils s'installaient pendant deux à trois ans à un endroit où ils avaient recours à la culture sur brûlis. Ils brûlaient ainsi une partie de la forêt pour fertiliser les sols dans le but d'y cultiver des ressources alimentaires et médicinales. Un territoire de chasse s'étendait autour de cette zone de culture. Dès que la fertilité de la terre diminuait, les Quichuas se déplaçaient vers un autre endroit. L'espace défriché avait ensuite le temps de se régénérer. La confrontation avec des expéditions de missionnaires à l'origine de la création de villages dès le $\mathrm{XVII}^{\mathrm{e}}$ siècle, puis des activités d'extraction de l'or et du caoutchouc entre 1890 et 1930, ont contraint les Quichuas à se sédentariser. Mais c'est particulièrement à partir des années 1950 que le processus s'est accéléré avec l'arrivée de la prospection et exploitation pétrolières accompagnées de politiques d'intégration du territoire amazonien, jusqu'ici délaissé, au reste de l'Équateur (UQUILLAS 1985 : 93110).

Les nombreux changements entrainés par le passage d'une vie nomade à une vie sédentaire ont eu une incidence sur la santé mentale autochtone (MAGORD 2005 : 247-262). Mais quelles sont les ruptures et continuités dans les pratiques culturelles que ces changements ont provoquées ? Comment expliquer que les Inuit du Nunavut, en dehors de l'aspect plus récent du processus de sédentarisation, connaissent un taux de suicide si élevé ?

Suite à leur sédentarisation, les Quichuas ont pu continuer à pratiquer la culture sur brûlis et l'agroforesterie. Si la première participe de nos jours à la déforestation - les populations ne se déplaçant plus d'un endroit à un autre, la forêt n'a plus le temps de se régénérer - les deux permettent néanmoins de faire perdurer un élément culturel caractéristique des Quichuas. Ces pratiques culturelles sont liées à des savoirs et savoir-faire pouvant encore se transmettre de génération en génération.

Les Inuit, en revanche, ont vu leur pratique culturelle de la chasse diminuer fortement. Les communautés instaurées par les programmes canadiens ont souvent été implantées loin des lieux de chasse et de migration d'animaux. Les déplacements forcés ont en outre eu des impacts sur les relations familiales, les relations matrimoniales et les personnes (DUPRE 2011 : 139-150). La pratique culturelle de la chasse, bien qu'encore possible, est de plus en plus difficilement réalisable. Les transmissions de savoir et 


\section{Simon FLEURY}

savoir-faire ont moins d'occasions de se réaliser par l'expérience d'une pratique culturelle . $^{8}$

Un deuxième élément significatif est le logement. La pratique culturelle de construction d'igloos tend également à disparaître pour laisser place à des logements fabriqués et financés par des fonds fédéraux canadiens. Les Quichuas du Napo bénéficient également de financements gouvernementaux ${ }^{9}$. Cependant, les investigations menées sur le terrain entre 2010 et 2013 ont montré que la plupart du temps, les Quichuas bénéficiaires de maisons « en dur », c'est-à-dire en béton, construisent juste à côté une maison en bois, selon leurs connaissances et savoir-faire propres ${ }^{10}$. Certains n'habitent même que cette dernière, se servant de la maison financée par le gouvernement pour entreposer du matériel. Les pratiques culturelles liées à la construction d'un logement se transmettent donc encore de génération en génération chez les Quichuas du Napo, quand chez les Inuit elles ne s'opèrent plus, ou moins, dans ce domaine.

La sédentarisation n'aurait donc pas coupé les Quichuas de leur environnement autant que les Inuit. Il en serait de même pour la pratique culturelle de la construction d'un logement. Les deux éléments soulevés favorisent la transmission d'un savoir entre générations et le développement d'un attachement " sécure » chez les enfants (CYRULKIK et JORLAN 2014). Il semble que chez les Quichuas du Napo, la sédentarisation a perturbé l'équilibre environnemental ${ }^{11}$ mais que l'adhésion à des normes sociales et le respect des obligations morales connaissent une continuité ; chez les Inuit, les trois semblent ébranlés.

Cela expliquerait en partie pourquoi, malgré une reconnaissance plus aboutie, les Inuit connaitraient d'avantages de problèmes de santé mentale. Ces constatations ne constituent pas des résultats sinon des pistes de réflexion que

\footnotetext{
${ }^{8}$ Inuit Qaujimajatuqangit: le rôle du savoir autochtone pour favoriser le bien-être des communautés inuites du Nunavut (Centre de Collaboration Nationale de la Santé Autochtone 2009-2010) (Shirley Tagalik, chercheuse en chef, Inukpaujaq Consulting)

${ }^{9}$ Cf Ministerio de desarrollo Urbano y Vivienda (MIDUVI)

${ }^{10}$ Nous ne prétendons pas que ces maisons soient faites selon une logique « ancestrale », celles-ci ayant la plupart du temps un toit en zinc, mais mettons en avant les savoirs requis pour les construire.

${ }^{11}$ Plus que la sédentarisation, ce sont l'urbanisation et la déforestation qui sont à l'origine aujourd'hui de nombreux changements dans l'organisation sociale (entretien personnel avec Françoise Morin). Les activités d'extraction minière, pétrolière, l'élevage de bovins et l'exploitation du bois sont les principales causes de la déforestation.
} 
la recherche entreprise souhaite approfondir. Nous avons constaté l'importance des pratiques culturelles et de la transmission du savoir et savoir-faire entre générations. Il convient alors de s'intéresser aux traumatismes intergénérationnels générés par les chocs biologique, structurel et identitaire décrits plus hauts, pour comprendre l'état de la santé mentale autochtone au Nunavut et en Amazonie équatorienne, dans un contexte de mondialisation.

\section{Ruptures et traumatismes intergénérationnels}

Jack Hicks, ancien conseiller pour la prévention des suicides auprès du gouvernement du Nunavut et chercheur statisticien sur le suicide, étudie l'évolution du taux de suicide chez les Inuit du Canada. Ses recherches ont permis de montrer comment le taux de suicide a évolué sur le temps, au Nunavut, mettant en exergue un pic au début des années 2000 chez les jeunes âgés de 15 à 24 ans. Il a également observé une augmentation significative du taux de suicide ces dernières années chez les Nunavummiut âgés de 25 à 34 ans ${ }^{12}$. Les raisons pour lesquelles une personne s'enlève la vie sont toujours complexes et multi-causales. Selon Hicks, les pics de suicide identifiés à partir des années 2000 correspondraient aux premières générations d'Inuit dont les parents ont été déplacés dans de nouveaux camps. Cette génération d'Inuit aurait ainsi été affectée par un traumatisme intergénérationnel traversé par leurs parents et grands-parents à cause, entre autres, des écoles résidentielles, de l'abattage de chiens ${ }^{13}$, ou encore du déplacement de population. En d'autres termes, ces générations où les pics de taux de suicide sont les plus importants évoluent au sein de familles où les pères continuent à souffrir de douleurs et colères persistantes.

La pratique culturelle permet de vivre une expérience culturelle. Au travers de cette dernière, des notions véhiculées par la langue et l'apprentissage de connaissances sont transmises d'une génération à l'autre. Ces transmissions permettent d'humaniser les enfants et de les rendre « aptes » ${ }^{14}$ à se débrouiller dans leur environnement.

\footnotetext{
12 "Nunavut's youth suicide epidemic" in "Who is next? How do we stop this?" " de Sandro Contenta. (article en ligne sur www.thestar.com).

${ }^{13}$ Lors du relogement des Inuit à partir des années 1950, la PCNO (Police à Cheval du Nord-Ouest) a reçu l'ordre d'abattre les chiens de traineau des Inuit qui étaient dépendants de ces animaux pour leurs déplacements et pour chasser. Cette politique aura eu des conséquences désastreuses.

${ }^{14}$ Terme employé dans le « inuit Qaujimajatuqangit » rédigé par Shirley Tagalik, chercheuse en chef, Inukpaujaq Consulting.
} 


\section{Simon FLEURY}

Les politiques actuelles de reconnaissance ne semblent pas comprendre ou prendre en considération la construction de l'humanité opérée grâce à la socialisation des enfants par leurs parents. Les expériences culturelles étant de plus en plus rares, le lien de socialisation et donc l'attachement sécure entre parents et enfants se détériorent. D'un côté, les parents porteurs d'un savoir et d'un mode de vie ne sont pas en mesure de répondre à une compréhension des forces globalisantes et de l'autre, les jeunes sont coupés de cette expérience culturelle. Le lien de continuité entre générations étant rompu, les processus de socialisation permettant de créer une capacité de résilience face aux traumatismes n'ont plus l'espace et le temps de s'accomplir. Au niveau individuel, la conscience sociale s'acquiert au travers du processus de socialisation. Toutefois ce processus est interrompu et parfois impossible dès lors que les agents socialisateurs (parents, enseignants, soignants) se sentent inutiles et en inadéquation, et quand les normes sociales sont discréditées (WESLEY-ESQUIMAUX: 2007).

Ainsi, ce que les Inuit appellent « les questions de la vie» ne sont plus abordées et plus maîtrisées. Il n'existe plus de lieu pour la culture, ciment de l'harmonie et de l'équilibre. Les pères comme les nouvelles générations sont confrontés à des situations qui ne favorisent pas la prise de confiance en soi et en sa culture. De surcroît, les trois chocs biologique, structurel et identitaire, le traumatisme intergénérationnel qu'ils ont engendré et les nouvelles mutations liées à la mondialisation rendent la définition, au niveau individuel comme collectif de ce qu'est la culture inuit, difficile.

Pourtant, d'après les recherches de Donald Taylor, c'est la capacité à pouvoir se définir qui est préalable à une bonne image de soi (TAYLOR 1997 : 74-190). Comment être fier de ce que l'on est si on ne sait pas qui on est? Comme le précise Tousignant, «c'est donc la confusion et l'aliénation qui s'ensuivent qui empêchent tout processus de résilience de prendre forme » (TOUSIGNANT, 2012: 145). Le bien-être lié à une santé mentale stable ne peut donc s'atteindre sans la capacité à se définir soi, c'est-à-dire son identité. Les Inuit touchés par les taux de suicide vivraient une crise identitaire telle qu'elle mènerait aux gestes les plus lourds de conséquences.

Dans le contexte des Quichuas du Napo, qui connaissent une mobilité favorisée par une distance moindre entre communautés et villes par rapport aux Inuit du Nunavut où les différents villages ne sont reliés que par internet et avions, une double vie ville-forêt se met en place. Les nouvelles générations issues de communautés vont généralement en ville pour accéder au système 
scolaire. Dès lors, une dichotomie entre vie familiale signifiant vie en forêt et vie scolaire signifiant accès à un nouveau mode de vie se met en place. Une observation de terrain le long du fleuve Napo en avril-mai 2014 a permis d'être confronté à un conflit intergénérationnel. Des parents reprochaient à leur fille de ne plus vouloir participer à la vie familiale et communautaire. L'intervention d'une guérisseuse aura même été demandée, afin de «retrouver l'harmonie familiale $»^{15}$. Il n'est pas question ici de traumatisme mais de décalage entre les vécus venant perturber la socialisation et amenant à une sensation de désolidarisation entre les générations. La rupture n'est pas brutale mais plus subtile et dénote chez les nouvelles générations un désintérêt pour les pratiques culturelles familiales.

L'examen des mécanismes du traumatisme intergénérationnel a permis de montrer dans quelle mesure celui-ci peut représenter un frein au processus de résilience. De plus, il semble que le décalage entre générations à l'origine des transformations des modes de vie semble accentuer une perte de cohésion. D’autres limites sont posées par les programmes de reconnaissance.

\section{Les limites des programmes de reconnaissance dans l'optique d'une résilience}

Les relations de pouvoir entre groupes minoritaire et majoritaire sont un facteur déterminant dans le processus de résilience employé par un groupe social, pour se remettre d'un traumatisme. Dans le contexte autochtone, l'existence d'une structure dominante majoritaire et les rapports de pouvoir qui en découlent, déterminent les limites d'une reconnaissance. La reconnaissance a lieu au sein d'un système centralisé qui est imposé, c'est-à-dire qu'elle offre dans une certaine mesure le droit aux populations autochtones de se créer une place dans une structure plus globale, mais n'offre pas la possibilité de la modifier en profondeur.

La reconnaissance s'effectue dans un rapport de pouvoir entre dominants et dominés. Elle ne peut donc pas être complète, dans la mesure où le groupe dominant se réserve l'ascendant et protège son hégémonie en s'assurant notamment l'accès à des ressources sur les territoires concernés. Ce rapport de force va dans le sens d'André Légaré (2009) et Cécile Pelaudeix (2008) qui soulèvent la possibilité que le Nunavut n'ait été créé que dans un souci de stratégie internationale, c'est-à-dire pour assurer la souveraineté

\footnotetext{
${ }^{15}$ Témoignage recueilli auprès de Juan Garces, le père de famille en mai 2015.
} 


\section{Simon FLEURY}

canadienne sur le territoire arctique face aux prétentions des États-Unis et de la Russie.

C'est ainsi que les quotas de chasse et de pêche imposés par le gouvernement du Nunavut sont perçus par de nombreux Inuit comme un moyen de les empêcher de pratiquer une activité culturelle importante dans la définition de leur identité. Or, certains témoignages montrent que les Inuit considèrent l'activité de la chasse et de la pêche importantes pour leur bienêtre $^{16}$. Dès lors, ces relations de pouvoir se retrouvent dans les politiques de reconnaissance en tant que forces hétéronomisantes, c'est-à-dire contraires à l'objectif d'autonomisation poursuivi. Elles ne participent pas à l'épanouissement du groupe qui n'a d'autre choix que de s'adapter par des stratégies d'appropriation des règles imposées ou par une opposition et une résistance. Ces éléments soulignent «certaines inadéquations entre le système mis en place au Nunavut et les buts recherchés par les Inuits, tels que formulés dans le préambule de l'Accord sur les droits territoriaux » (MAGORD 2005 : 249).

Les forces hétéronomisantes ne favorisent pas la réalisation d'expériences et de pratiques culturelles. Les pratiques non adaptées à la nouvelle forme de gouvernance mise en place grâce aux politiques de reconnaissance, selon un modèle extérieur, ne survivent pas. La socialisation de l'individu (inunnguinik ${ }^{17}$ ), c'est-à-dire la transmission de génération en génération de valeurs et connaissances culturelles au travers de l'expérience culturelle, ne s'insère pas dans la forme institutionnalisée de la reconnaissance. Il faut au contraire former les adultes pour qu'ils puissent former les jeunes générations à la gestion d'institutions formalisées, selon la logique culturelle du dominant, ce qui créé un nouveau besoin : s'approprier une logique culturelle extérieure. Ce besoin s'explique par une institutionnalisation rapide du Nunavut, qui accapare la presque totalité de l'énergie du nouveau gouvernement, qui tente de mettre en place «l'énorme machine administrative par laquelle les directives et les subsides de l'État transitent » (MAGORD 2005: 258). Le système administratif ressemble plus à une forme de globalisation de la gestion sociétale qu'à un outil d'autonomisation adapté au monde inuit.

\footnotetext{
${ }^{16}$ Pauktuutit Inuit Women of Canada, RASMUSSEN Derek, GUILLOU, Jessica : Developing an inuit specific framework for culturally relevant health indicators incorporating gender-based analysis - Journal de la santé autochtone, Organisation Nationale de la Santé Autochtone (mars 2012) p. 24-35.

${ }^{17}$ Terme inuit pour « socialisation » employé dans le Inuit Qaujimajatuqangit.
} 
L'implantation d'un système de gouvernance non adéquat au monde inuit ajoute à la perte des repères d'origine une sensation de non maîtrise de son destin. Les trois chocs identifiés plus haut semblent donc se diluer et se perpétuer dans le temps. Cela génère des frustrations et un immobilisme pouvant accentuer les problèmes d'addiction et de santé mentale.

\section{Conclusion : la culture et les liens familiaux comme source de résilience}

Les trois chocs, biologique, structurel et identitaire ont généré chez les populations autochtones des souffrances et une «blessure de l'âme » ${ }^{18}$ qui, à l'heure actuelle, font de la période coloniale un déterminant social de leur santé mentale.

La résilience, entendue comme un processus permettant de rebondir suite à un traumatisme, diffère en fonction des groupes autochtones. L'expérience du Nunavut montre que la création d'un territoire avec un gouvernement public au service des Inuit n'est pas une garantie suffisante pour assurer la santé mentale et le bien-être individuel et collectif.

Dans les deux cas, les stratégies qui permettent une continuité dans les pratiques culturelles, participent au maintien d'une santé mentale et d'un bienêtre. L'entretien des pratiques culturelles sont rendues possibles grâce à l'expérience culturelle partagée entre générations. Les Inuit considèrent que pour devenir un être humain, il faut atteindre une maîtrise élevée des « questions de la vie » en plus de compétences permettant l'autonomie dans le savoir-faire. Chaque famille doit identifier les domaines dans lesquels leurs enfants pourront exceller (construire un igloo, chasser) pour les accompagner sur cette voie. ${ }^{19}$ Un renforcement des liens intergénérationnels doit permettre par conséquent une meilleure résilience grâce à la transmission de savoir et de savoir-faire lors d'expériences culturelles. Or, dans le cas Inuit, un traumatisme intergénérationnel coupe les plus jeunes d'un dialogue avec les anciens et chez les Quichuas, la dichotomie ville-forêt vécue par les nouvelles générations les oriente davantage vers les villes, créant un décalage entre générations.

Dès lors, les programmes de reconnaissance devraient prendre en compte le culturel et la résilience du traumatisme colonial dans leur élaboration

\footnotetext{
${ }^{18}$ Idem

${ }^{19}$ Terme Inuit Qaujimajatuqangit
} 


\section{Simon FLEURY}

et leur mise en place. Tant que les structures de la reconnaissance seront pensées selon un système étranger aux pratiques culturelles autochtones, elles exerceront une force hétéronomisante au sein du processus d'autonomisation et ne participeront pas à une résilience. Le Qaujimajatuqangit (IQ) et le Sumak Kausay (SK) sont des propositions émanant des élites Inuit et Quichuas (HIDALGO-CAPITAN, GUILLEN GARCIA, DELEG GUAZHA 2014). Ces concepts reflètent deux visions autochtones de la société selon deux contextes et parcours historiques différents. Ils pourraient être pris en compte aussi bien d'un point de vue théorique que pratique pour ainsi intégrer le culturel et l'importance de l'harmonie familiale au système de gouvernance autochtone.

Cependant, ils sont encore confrontés à la « soi-disant suprématie de la connaissance scientifique et l'hypothèse implicite que d'autres connaissances [et pratiques médicales] sont inefficaces » (NELSON 2011:6 ${ }^{20}$. Le projet de recherche propose de mettre en perspective $1^{\prime} \mathrm{IQ}^{21}$ et le $\mathrm{SK}^{22}$ en comparant les contextes et circonstances propres qui les ont vus naître pour mieux comprendre l'originalité de chacune de ces visions de la société dont le but est d'atteindre un bien-être social et culturel. Nous espérons ainsi participer à l'amélioration de l'intégration des visions autochtones du monde dans les recherches en santé mentale.

\section{Bibliographie}

ALDERETE, Ethel Wara (2004), Salud y Pueblos Indigenas, Quito, Ecuador, Ediciones Abya Yala.

BOUCHARD Gérard (2001), Genèse des nations et cultures du Nouveau Monde : essai d'histoire comparée, Québec, Edition Boréal, p. 37-75.

\footnotetext{
${ }^{20}$ NELSON Sarah (2011) « Remise en question des hypothèses cachées. Les normes coloniales en tant que déterminants de la santé mentale des autochtones », Rapport pour le Centre de Collaboration Nationale de la Santé Autochtone, Université du Nord de la Colombie Britannique.

${ }^{21}$ L'IQ est considéré par les leaders Inuit du Nunavut comme une technologie vivante. Il englobe les savoirs traditionnels qui correspondent à la vision inuit du monde et pouvant avoir une utilité aujourd'hui pour répondre aux problèmes que rencontre la société inuit du Nunavut. Il s'agit d'un moyen de rationaliser la pensée et l'action, d'organiser des tâches et des ressources, d'organiser la famille et la société en des tous cohérents (Jaypeetee Arnakuk).

${ }^{22}$ Le SK est un concept qui a d'abord vu le jour dans les Andes, suite au travail de penseurs autochtones et indigénistes. Il peut se traduire par «Bien Vivre ». Il propose de revoir la place de l'homme dans l'environnement et de repenser l'organisation de la société en réponse aux limites de la civilisation occidentale. Ce concept est un nouveau paradigme de civilisation.
} 
CHACHAMOVICH, Eduardo, HAGGARTY, Jack, CARGO, Margaret, HICKS, Jack, KIRMAYER, Laurence J. and TURECKI, Gustavo (2013), "A psychological autopsy study of suicide among Inuit in Nunavut: methodological and ethical considerations, feasibility and acceptability", International Journal of Circumpolar Health, 72: 20078, http://dx.doi.org/10.3402/ijch.v72i0.20078

CYRULNIK Boris et Gérard JORLAND dir. (2012), Résilience, Connaissances de base, Paris, Editions Odile Jacob.

DELÂGE Denys et TRUDEL François (1991), « Présentation. La rencontre des deux mondes », Anthropologie et sociétés, vol. 15, n ${ }^{\circ}$, p. 5-12.

DUPRÉ Florence (2011), “'South camp was our home’: le déplacement forcé des Inuit des îles Belcher (Nunavut)», Recherches amérindiennes au Québec, vol. 41, n²-3, p. 139-150.

DURAN, E. et DURAN, B. (1995). Native American Postcolonial Psychology. Albany, NY: State University of New York Press.

GOEBERT Deborah (2014), "A concise guide to understanding suicide", in Epidemiology, Pathophysiology, and Prevention, Koslow S.H., Ruiz P. and Nemeroff C.B. eds, Cambridge, Cambridge University Press, p. 159-165

HICKS Jack (2007), "The social determinants of elevated rates of suicide among Inuit youth", Social Suffering, International Workgroup for Indigenous Affairs, Copenhagen, p. 3-4

HIDALGO-CAPITAN Antonio Luis, GUILLEN GARCIA Alejandro et DELEG GUAZHA Nancy (2014), "Antología del Pensamiento indigenista Ecuatoriano sobre Sumak Kausay”, Sumak Kausay Yuyay, Quito, Eds Huelva y Cuenca.

LACROIX Laurent (2013), Un multiculturalisme sans minorités ? Quelques réflexions sur l'Etat plurinational en Bolivie et en Equateur », Belgeo, Revue belge de géographie, 3 | 2013, consulté le 03 novembre 2014. http://belgeo.revues.org/11512

LÉGARÉ André (2009), «Nunavut : entre le rêve et la réalité. Bilan de dix années d'autonomie gouvernementale inuite et prospection socioéconomique», Journal of Canadian Studies/Revue d'études canadiennes, Volume 43, N², Juin 2009, p. 23-56.

LOPERA VALLE Johan Sebastián et ROJAS JÍMENEZ Sara (2012), "Salud mental en poblaciones indígenas. Una aproximación a la problemática de salud pública", Medicina UPB, Universidad Pontificia Bolivariana, Medellin, Colombie, vol 31, numéro 1, 2012. 


\section{Simon FLEURY}

MAGORD André (2005), «Identité et politique au Nunavut. Eléments de réflexion critique », in Petites sociétés et minorités nationales, sous la direction de Jacques L. Boucher et Joseph Yvon Thériault, Québec, Presses de l’Université du Québec, p. 246-262.

--- (2014) : « Minorités historiques, autonomie et néo-libéralisme au Canada », Mémoire(s), identité(s), marginalité(s) dans le monde occidental contemporain, 11 | 2014, consulté le 28 juillet 2016. http://mimmoc.revues.org/1576

MARIN WEXLER Lisa (2006), "Inupiat youth suicide and culture loss: changing community conversations for prevention", Elsevier, Social science \& Medicine 63 (2006) 2938-2948, Maniilaq Association Heath, MA, USA Available online 6 September 2006.

MARTÍNEZ SASTRE Javier (2014), "Paraíso en Venta: Desarrollo, etnicidad y ambientalismo en la frontera sur del Yasuní (amazonia ecuatoriana)". Tesis con mención de Doctorado Internacional. Universitat de Lleida.

MURATORIO Blanca(1998), Rucuyaya Alonso y la historia social y económica del Alto Napo 1850-1950, Quito, Editions Abya Yala.

PELAUDEIX Cécile (2008), «Culture de l'Arctique, Identité nationale et enjeux circumpolaire », in La société canadienne en débats - What holds Canada together?, Alain Faure et Robert Griffiths dir., Paris, L'Harmattan, p. 161-176.

TAYLOR, Donald M. (1997), « The quest for collective identity: the plight of disadvantaged ethnic minorities », Canadian Psychology/Psychologie Canadienne, 38 (3), p. 74-190.

TAGALIK Shirley, "Inuit Qaujimajatuqangit : le rôle du savoir autochtone pour favoriser le bien-être des communautés inuites du Nunavut », Centre de Collaboration Nationale de la Santé Autochtone.

UQUILLAS Jorge E. (1985), «Expansion du front pionnier et Amérindiens en Amazonie équatorienne », Actes du séminaire des 15 et 16 octobre 1985 à Paris «Connaissances du milieu amazonien », Paris, Editions de l'Orstom, p. 93-110.

WESLEY-ESQUIMAUX, Cynthia C. (2007), "The intergenerationnal transmission of historic trauma and grief", Social Suffering, International Workgroup for Indigenous affairs, Copenhagen, July 2007, p. 6-11. 